\title{
Identification of quantitative trait nucleotides and candidate genes for soybean seed weight by multiple models of genome-wide association study
}

Benjamin Karikari, Zili Wang, Yilan Zhou, Wenliang Yan, Jianying Feng ${ }^{*}$ (ID and Tuanjie Zhao

\begin{abstract}
Background: Seed weight is a complex yield-related trait with a lot of quantitative trait loci (QTL) reported through linkage mapping studies. Integration of QTL from linkage mapping into breeding program is challenging due to numerous limitations, therefore, Genome-wide association study (GWAS) provides more precise location of QTL due to higher resolution and diverse genetic diversity in un-related individuals.

Results: The present study utilized 573 breeding lines population with 61,166 single nucleotide polymorphisms (SNPs) to identify quantitative trait nucleotides (QTNs) and candidate genes for seed weight in Chinese summersowing soybean. GWAS was conducted with two single-locus models (SLMS) and six multi-locus models (MLMS). Thirty-nine SNPs were detected by the two SLMs while 209 SNPs were detected by the six MLMs. In all, two hundred and thirty-one QTNs were found to be associated with seed weight in YHSBLP with various effects. Out of these, seventy SNPs were concurrently detected by both SLMs and MLMs on 8 chromosomes. Ninety-four QTNs colocalized with previously reported QTL/QTN by linkage/association mapping studies. A total of 36 candidate genes were predicted. Out of these candidate genes, four hub genes (Glyma06g44510, Glyma08g06420, Glyma12g33280 and Glyma19g28070) were identified by the integration of co-expression network. Among them, three were relatively expressed higher in the high HSW genotypes at R5 stage compared with low HSW genotypes except Glyma12g33280. Our results show that using more models especially MLMs are effective to find important QTNs, and the identified HSW QTNs/genes could be utilized in molecular breeding work for soybean seed weight and yield.

Conclusion: Application of two single-locus plus six multi-locus models of GWAS identified 231 QTNs. Four hub genes (Glyma06g44510, Glyma08g06420, Glyma12g33280 \& Glyma19g28070) detected via integration of cOexpression network among the predicted candidate genes.
\end{abstract}

Keywords: Association mapping, Complex nature, Haplotype, Hub genes, Co-expression network

\footnotetext{
* Correspondence: fengjianying@njau.edu.cn; tjzhao@njau.edu.cn

National Center for Soybean Improvement, Key Laboratory of Biology and

Genetic Improvement of Soybean (Ministry of Agriculture), State Key

Laboratory of Crop Genetics and Germplasm Enhancement, Nanjing

Agricultural University, Nanjing 210095, People's Republic of China
}

(c) The Author(s). 2020 Open Access This article is licensed under a Creative Commons Attribution 4.0 International License, which permits use, sharing, adaptation, distribution and reproduction in any medium or format, as long as you give appropriate credit to the original author(s) and the source, provide a link to the Creative Commons licence, and indicate if changes were made. The images or other third party material in this article are included in the article's Creative Commons licence, unless indicated otherwise in a credit line to the material. If material is not included in the article's Creative Commons licence and your intended use is not permitted by statutory regulation or exceeds the permitted use, you will need to obtain permission directly from the copyright holder. To view a copy of this licence, visit http://creativecommons.org/licenses/by/4.0/ The Creative Commons Public Domain Dedication waiver (http://creativecommons.org/publicdomain/zero/1.0/) applies to the data made available in this article, unless otherwise stated in a credit line to the data. 


\section{Background}

The world's human population is estimated to reach 10 million by 30 -year time [1] with increasing abiotic and biotic stress as well as reduction in arable land for agricultural activities [2]. This implies that food and nutritional security is under threat. Legume crops including soybean (Glycine $\max$ L. Merr.) play a significant role in ensuring global food and nutritional security in addition to their abilities to improve soil quality through nitrogen fixation [3]. Consumption of legume crops is associated with health and physiological benefits like prevention of cardiovascular diseases, obesity, diabetes mellitus, cancer and relief of menopausal symptoms [4-6]. However, the insufficient soybean production in China and many under-developing countries is a big challenge and per unit yield of soybean needs to be improved rapidly.

One of the major determinants of soybean yield, seed use and evolutionary fitness is seed weight [7-9]. For example, large-seeded cultivars are used for boiled soybean (nimame), green soybean (edamame), soymilk and soybean curd (tofu), while small-seeded cultivars are suitable for fermented soybean (nattō) and sprout production [10-12]. Soybean breeders need to create big variation of seed weight for selection of varieties with different end-use purposes. Seed weight is also an important trait that was targeted during soybean domestication [13-16], and the range of 100-seed weight can vary from less than $1 g$ in wild soybean (Glycine soja Sieb. et Zucc.) accessions to more than $60 \mathrm{~g}$ in some specific edamame varieties.

As a complex quantitative trait, seed weight is assumed to be controlled by several major genes/loci plus numerous undetectable loci with minor effects (thus, polygenes), interacting with environments. More than 300 quantitative trait loci /nucleotides (QTL/QTNs) for soybean seed weight have been reported on SoyBase (www.soybase.org) via linkage mapping. However, integrating results from linkage mapping into breeding program is challenging due to the higher confidence interval and less genetic variation [17]. As a result in the recent years, marker-trait association is used to take advantage of all recombination events that occur in the evolutionary history of a natural population based on linkage disequilibrium (LD) [18, 19]. Marker-trait association allows researchers to utilize natural diversity and locate valuable genes in the genome [18]. For instance, Miao et al. [20] recently applied regional association mapping for seed oil and identified GmSWEET39 (Glyma.15 g049200/Glyma15g05470) which was subsequently overexpressed in Arabidopsis leading to at least $10 \%$ increase in seed oil content.

Tens of QTNs have been detected and reported through genome-wide association studies (GWAS) across the 20 chromosomes [13, 21-30]. However, different mapping results can be obtained due to population type, size and
GWAS method. Single-marker genome-wide scan models such as mixed linear model (MLM) and general linear model (GLM) are mostly frequently used in genetic dissection of soybean seed weight. These models have certain limitations including the issue of multiple test correction for threshold value of significance, and mapping power [31]. A number of multi-locus models have been developed and applied in recent GWAS in several crops including soybean. Six of such models (mrMLM, FASTmrMLM, FASTmrEMMA, pLARmEB, pKWmEB \& ISIS EMBLASSO) are implemented in $\mathrm{R}$ with the mrMLM.GUI package [32]. These models have become the state-of-theart procedure to identify QTNs with complex traits due to their detection power and robustness [33-37].

Bioinformatics tools have enhanced easy identification of potential genes for target QTL. One of the strategies is to utilize co-expression network which aims at prioritizing functionally related genes. It has been successfully used in several crops such as maize [38], rice [39], peanut [40], Arabidopsis [41], soybean [42, 43], among others. By integration of co-expression network analysis, a class of hub genes which induce major transcriptome reprogramming during grapevine development were identified Palumbo et al. [44]. The hub genes (genes highly connected) may give clue on the role of those genes in the network [45].

In most of the earlier reported GWAS for seed weight, population sizes were mostly $<500$ [29, 30, 46-48]. The population size, genetic diversity as well as genome coverage/number of SNPs, linkage disequilibrium, and statistical methods used have been reported to affect the power of GWAS [17, 49, 50]. Therefore, our present study utilized 573 breeding lines with 61,166 SNPs to conduct marker-trait association viz., two single-locus models (SLMs) and six multi-locus models (MLMs) to identify significant SNPs. Also, potential candidate genes were predicted, out of which hub genes were identified by the integration of functional co-expression network. Application of multiple models of GWAS detected 231 QTNs, out of which 94 co-localized with earlier reported QTL/QTNs. This demonstrate the use of multiple models of GWAS to unravel the complex architecture of seed weight in our recently developed diverse breeding lines.

\section{Results}

\section{Phenotypic variation of HSW in the YHSBLP}

The phenotypic variation of HSW in YHSBLP across the four environments (E1, E2, E3 and E4) followed a normal distribution, typical of quantitative traits (Fig. 1). In the E1, HSW ranged from 7.24-37.19g with the mean of $19.40 \pm 4.47 \mathrm{~g}$ whereas E2, E3 and E4 had a range (mean \pm standard deviation) of 8.23-39.70 g (21.22 $\pm 4.62 \mathrm{~g}), 7.71-$ $36.32 \mathrm{~g}(20.43 \pm 4.80 \mathrm{~g})$ and $8.38-36.78 \mathrm{~g}(20.09 \pm 4.78 \mathrm{~g})$, respectively (Additional File 1: Table S1). The HSW was significantly $(P<0.001)$ affected by genotype, environment 


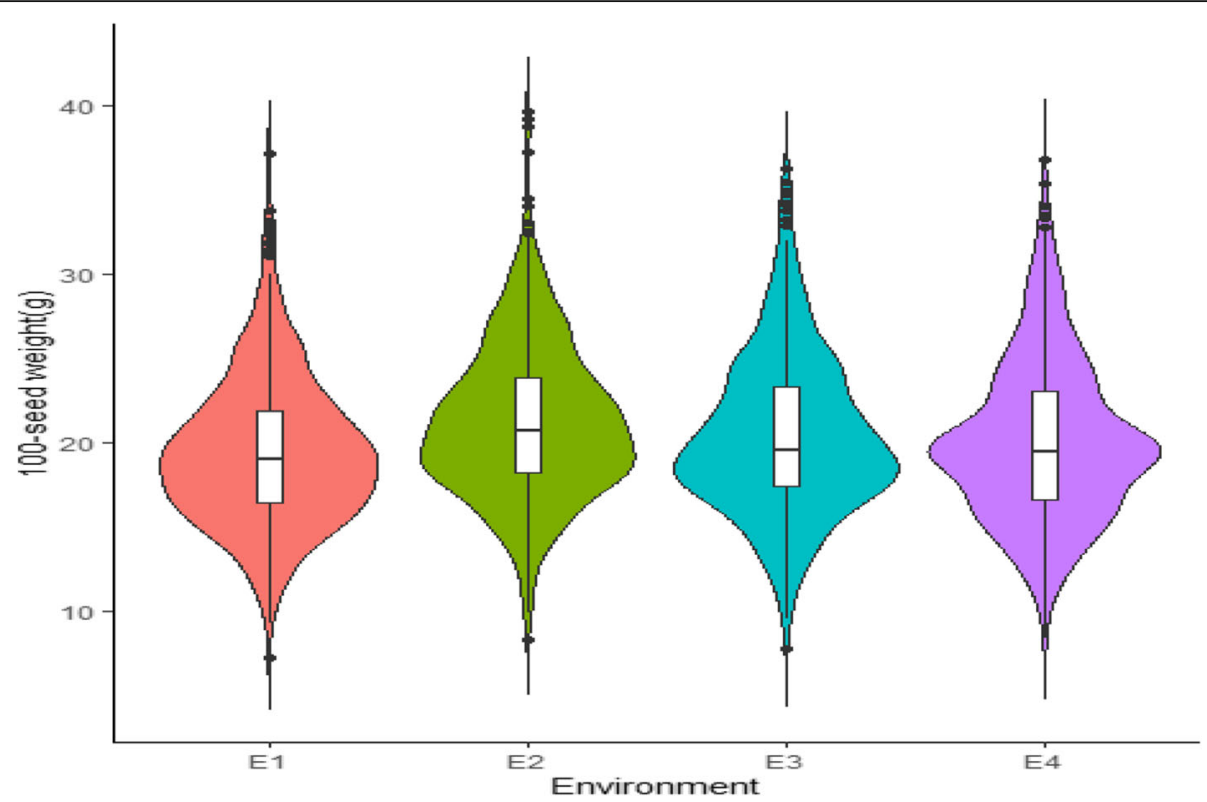

Fig. 1 Variation of HSW in each of the environments. The black in the middle of the box shows the median, the white box indicates the range from the lower quartile to the upper quartile, the black line represents the dispersion and frequency distribution of the phenotypic data. The black dots represent phenotypic data that were extreme in each environment

and genotype by environment interaction (Table 1). The broad-sense heritability $\left(h^{2}\right)$ was $98.53 \%$. These suggest that HSW in the summer sowing of YHSBLP was mainly influenced by genetic factors with less effect by environmental factors (Table 1).

SNPs distribution and population structure in the YHSBLP All the 573 accessions were genotyped by RAD-seq technology. After removing monomorphic markers as well as markers with $\mathrm{MAF}<5 \%$ and missing and heterozygous allele rate $>30 \%$, a total of 61,166 SNPs were used for this study. The total length of the genome was $950,068,807 \mathrm{bp}$ ( $950.07 \mathrm{Mb})$ representing $85.21 \%$ of the genome of soybean. The range of the number of SNPs per chromosome was 1467-4844 with chromosome 5 and 18 having the least and highest, respectively. The highest and lowest SNP density of 91.9 SNPs/Mb and $35 \mathrm{SNPs} / \mathrm{Mb}$ were found on chromosome (Chr.) 15 and Chr.05, respectively, with the longest chromosome being Chr.18 (Fig. 2).

The 573 accessions were grouped into three subpopulations as evident by population structure obtained from
ADMIXTURE software (Fig. 3a \& b), phylogenetic analysis (Fig. 3c) and PCA (Fig. 3d). The first two PC accounted for $22.60 \%$ variation (Fig. 3d). The lines with the probability $(\mathrm{Q})$ more than 0.70 score were considered as pure lines while those with $Q \leq 0.7$ were considered as admixtures. The subpopulation 1 comprised 107 pure lines with an average HSW of $17.68 \mathrm{~g}$. The subpopulation 2 and 3 consisted of 101 and 92 pure lines, respectively, with average HSW of $22.95 \mathrm{~g}$ and $19.73 \mathrm{~g}$ in that same order. The HSW differed significantly among the subpopulations. The remaining 273 admixtures had average HSW of $20.52 \mathrm{~g}$.

\section{SNP-trait association mapping}

A total of 39 SNPs significantly associated with HSW in at least one environment were detected via MLM $(\mathrm{K}+$ $\mathrm{Q})$ and CMLM (K+ PCA), respectively, with $-\log _{10}(P)=$ 4.00-12.25 (Additional File 2: Table S2; Additional File 3: Fig. S1; Additional File 4: Fig. S2). Among the 39 loci, twenty-four were detected by both models (MLM and CMLM) (Additional File 2: Table S2; Additional File 5: Fig. S3A). These QTNs were distributed unevenly on 11

Table 1 Joint ANOVA for HSW across the 4 environments

\begin{tabular}{lllllll}
\hline Source & DF & SS & MS & $F$ Value & $P$ value & $h^{2}$ \\
\hline Environment & 3 & $15,355.00$ & 5118.39 & 5.26 & 0.0261 & $<0.0001$ \\
Rep(Environment) & 8 & 7871.95 & 983.99 & 715.38 & $<0.0001$ \\
Genotype & 572 & $119,437.00$ & 208.81 & 17.64 & $<0.0001$ \\
GenotypexEnvironment & 1665 & $19,977.00$ & 12.00 & 8.72 & \\
Residual & 4228 & 5849.90 & 1.38 & & \\
\hline
\end{tabular}




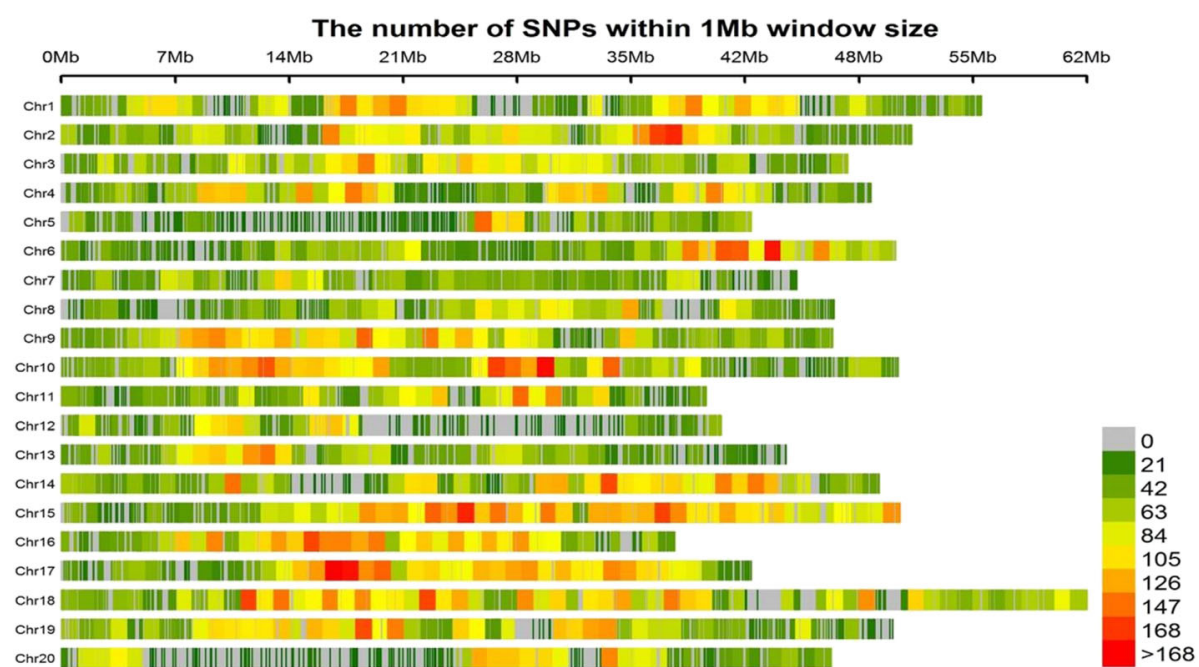

Fig. 2 Distribution of 61,166 SNPs on the 20 chromosomes of soybean. The horizontal axis shows chromosome length (Mb); the vertical axis gives the chromosome number and the different colors depict SNP density (the number of SNPs per window)

chromosomes. Each of the models (MLM and CMLM) detected the highest number of six QTNs on Chr.02 and Chr.13, respectively. Out of these QTNs, seven were detected in two environments by the two SLMs on five chromosomes. Chromosome 14 harbored three QTNs (qHSW-14-5, qHSW-14-10 \& $q H S W-14-11)$ whereas one QTN each was harbored on Chr08 (qHSW-8-8), Chr.13 (qHSW-13-26), Chr.15
(qHSW-15-4) and Chr.16 (qHSW-16-5) (Additional File 2: Table S2). The highest number of 16 QTNs were detected in environment (E4) by MLM whereas CMLM detected 12 QTNs in either E2 or E4 (Fig. 4). Most of the SNPs detected overlapped with QTL detected by earlier linkage mapping studies published on SoyBase and some association mapping studies (Additional File 2: Table S2).
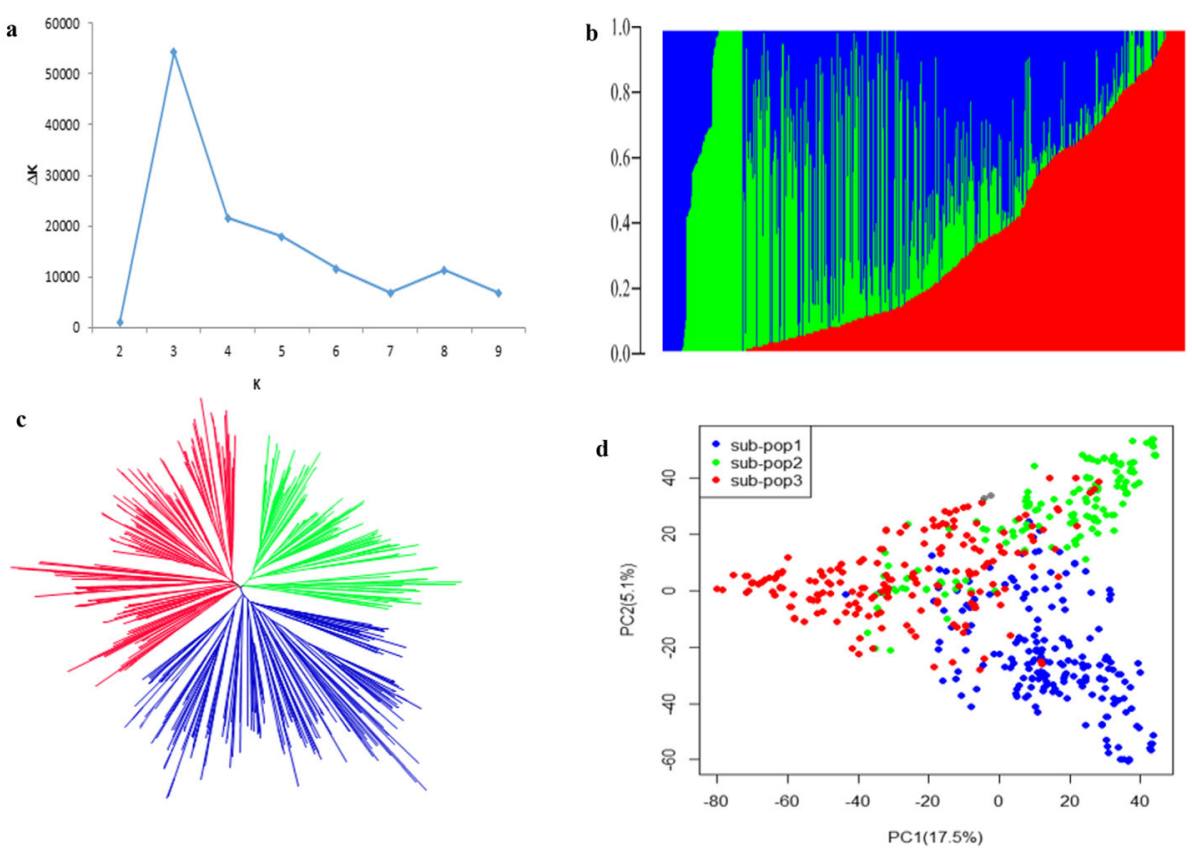

Fig. 3 Population structure of 573 accessions. a Plot of $\Delta K$ calculated for $K=1-10$. b Population structure obtained from ADMIXTURE software. Three colors (blue, green and red) represent three subpopulations. Each color represents one inferred ancestral population. Each vertical column represents one individual and colored segment in each column represents percentage of the individual inferred ancestral population in the YHSBLP. c A neighbor-joining tree of the YHSBLP with three clusters. d Principal Component Analysis plot 


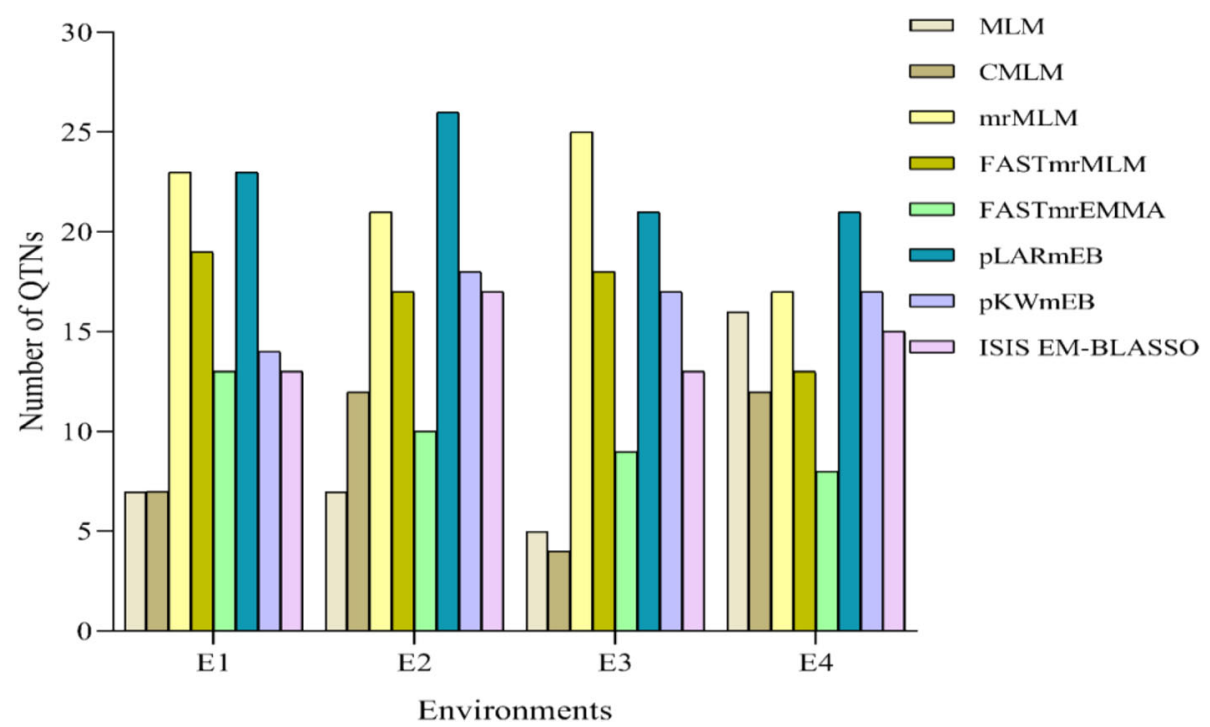

Fig. 4 Total number of SNPs detected by the 8 models in each environment. The X and Y-axis represent the environments (E1, E2, E3 \& E4) and number of SNPs detected, respectively

Six MLMs viz. mrMLM, FASTmrMLM, FASTEMMA, pLARmEB, pKWmEB and ISIS EM-BLASSO detected a total of 209 QTNs across all the 20 chromosomes unevenly with the range of logarithm of odd $(L O D)=3.01-$ 17.31 (Additional File 2: Table S2). The highest number of 13 QTNs were detected on Chr.13 by mrMLM whereas 25 QTNs were identified in E2 by pLARmEB (Fig. 4; Additional File 2: Table S2). The number of
QTNs detected by either of the six MLMs ranged 32-75, out of these, 11-44 QTNs were detected by at least two of the models concurrently (Additional File 2: Table S3). Out of the total 209 QTNs detected by six MLMs, thirty-nine were detected simultaneously in at least two environments with at least two models including qHSW-13-26 \& $q H S W-14-10$ (Additional File 2: Table S2).
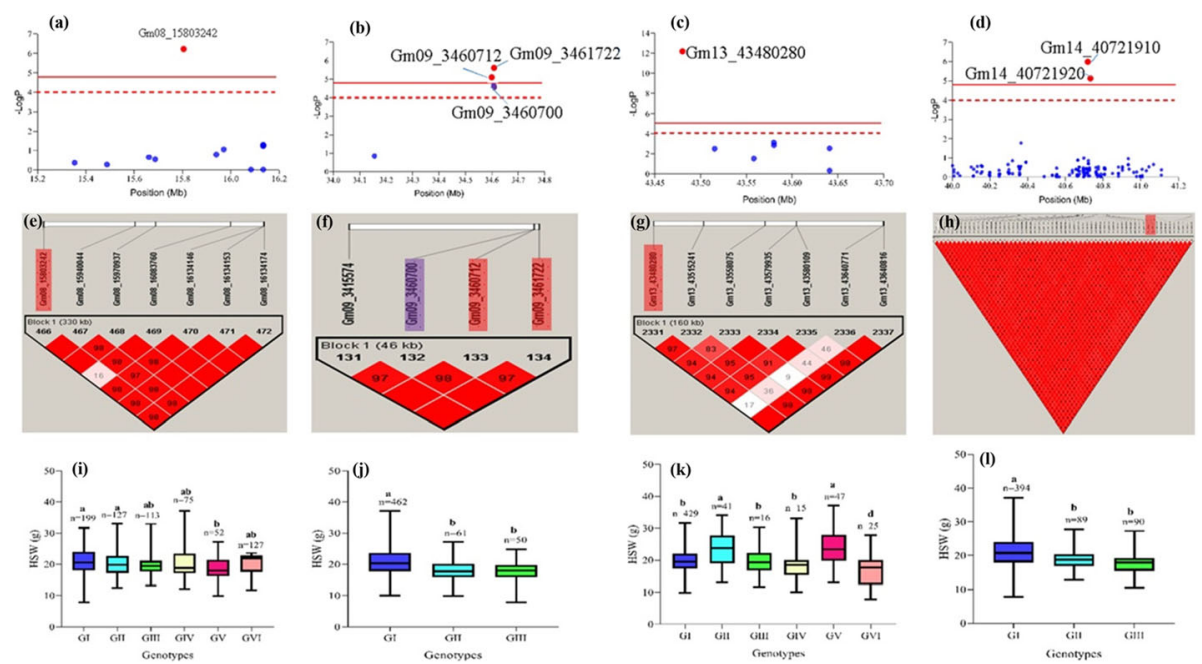

Fig. 5 Manhattan plots, haplotype block analyses of selected QTNs and allele effect on seed weight (boxplot). qHSW-8-8 are in a, e \& i. qHSW-9-4 are in $\mathbf{b}, \mathbf{f} \& \mathbf{j}$. $9 H S W-13-26$ are in $\mathbf{c}, \mathbf{g} \& \mathbf{k}$. GHSW-14-10 \& aHSW-14-11 are in $\mathbf{d}, \mathbf{h} \& \mathbf{I}$. The significant SNPs detected within each block are displayed in the Manhattan plots $(\mathbf{a}, \mathbf{b} \& \mathbf{c})$ and the dotted red line represents the reduced threshold (4) whereas the completed red line represents the Bonferroni correction threshold (4.79). The SNPs detected in each block are shaded red and purple for those that exceeded the 4.79 and 4 , respectively. The boxplot for each of the blocks were obtained by the average seed weight across the 4 environments (E1, E2, E3 \& E4) $(\mathrm{I}, \mathrm{J}, \mathrm{K} \& \mathrm{~L})$. The accessions were grouped and pairwise comparisons conducted by Duncan's Multiple Range Test at $P<0.05$. The boxes with a common alphabet indicate no significant difference in seed weight. Number of accessions (n) in each sub-class is represented on top of each box. GI-GVI represents a number of groupings of the 573 accessions in each block 
A total of 231 QTNs were identified by the two SLMs plus the six MLMs, out of these, seventeen were detected concurrently by the two categories of models (Additional File 2: Table S2; Additional File 5: Fig. S3B). $q H S W-2-1, q H S W-2-8$, $q H S W-2-10, q H S W-9-12, q H S W-13-8, q H S W-13-26, q H S W-$ 14-10, qHSW-14-12 \& qHSW-17-4 were detected by more than of two the MLMs in at least three environments as well as by at least one of the SLMs in one environment (Additional File 2: Table S2). Pairwise comparison among the eight models showed vary range of QTNs (2-44) detection concurrently (Additional File 5: Fig. S3).

\section{Allele effect of QTNs on seed weight}

Haplotype block analyses were conducted for $q H S W-8-8$ (Gm08_15803242), qHSW-9-4 (Gm09_3461722), qHSW13-26 (Gm13_43480280) and two tightly linked SNPs, Gm14_40721910 (qHSW-14-10) \& Gm14_40721920 (qHSW-14-11) in Haploview software with four gamete rule method (Fig. 5a-l). The distance within each block ranged 46-490 kb with range of 4-97 SNPs. The 573 accessions were grouped into 3-6 categories in each block with significant variation in seed weight.

\section{Candidate genes prediction and further analyses}

Potential candidate genes were mined from $500 \mathrm{~kb}$ upstream and downstream of significant SNPs that were detected in at least two environments. SNPs within a block that were detected in at least one environment, only one was used to identify candidate genes. In all, thirty-six candidate genes were identified using the orthologs in Arabidopsis of which 14 and 22 were located upstream and downstream of the SNPs positions, respectively (Additional File 6: Table S4). Five SNPs had two potential candidate genes each, for instance, SNP at Gm08_15803242 (qHSW-8-8) had Glyma08g20770 and Glyma08g20780 at 38.65 and $27.86 \mathrm{~kb}$ downstream. These two genes code for ATP binding cassette (ABC) transporter protein with biological function of transport; transmembrane transport which have been demonstrated to play significant role in regulating seed size/weight with effect on seed yield [51]. These two genes together with Glyma06g17520, Glyma06g44510, Glyma09g04840, Glyma11g27070, Glyma12g33280, Glyma13g18280 and Glyma13g17890 are annotated to be involved in sugar/sucrose/ monosaccharide transport. The remaining 25 genes are involved in two or more biological processes such as cell proliferation, regulation of cell size, cell wall modification, flower/its part development, seed development, seed coat development and other biological processes which play key roles in regulating seed size/weight (Additional File 6: Table S4). From the RNA-Seq Atlas developed by Severin et al. [52] available on SoyBase database, it was discovered that all the predicted candidate genes are highly expressed in seedrelated tissues as well as seed developmental stages except Glyma09g24020 (Additional File 6: Table S5).
The $1.5 \mathrm{~kb}$ upstream of each candidate gene was explored for seed-related regulatory elements. Three seedrelated cis-elements (GCN4_motif, MSA-like and RYelement) were identified in the promoter regions of 13 of the predicted candidate genes (Additional File 6: Table S6). GCN4_motif [TGAGTCA] which is involved in endosperm expression was found in Glyma01g38450, Glyma04g06760, Glyma13g17890, Glyma15g17040, Glyma15g39730 and Glyma19g28070 [53]. MSA-like [(T/ C)C(T/C)AACGG(T/C)(T/C)A] element which is involved in cell cycle regulation was found in Glyma02g07240, Glyma09g04840 and Glyma14g11930 [54]. RY-element [CATGCATG] involved in seed-specific regulation was identified in Glyma03g06420, Glyma03g06600, Glyma06g17520 and Glyma12g33280 [55]. Plant growth and development are regulated by circadian related genes especially flowering time. In addition, the circadian clock [CAAAGATATC] was detected in Glyma01g38450, Glyma04g06760, Glyma06g44510, Glyma07g11550, Glyma08g20780 and Glyma13g17890. A study conducted by Hudson [56] demonstrated that the circadian clock controlled transcriptome of developing soybean seeds. Twenty of the predicted candidate genes had ABRE element involved in the abscisic acid responsiveness which have been reported to play a primary role in seed maturation [57].

To understand possible interaction among the 36 candidate genes whilst mining for other genes, the predicted candidate genes were subjected to SoyNet which has 40,182 soybean genes ( $73 \%$ of the coding genome) with two million functional network in soybean [58]. A dense interaction network among 213 genes distributed across the 20 chromosomes were found comprising 15 of the predicted genes in this study (Additional File 7: Fig. S4A\&B). Four hub genes (Glyma06g44510, Glyma08g06420, Glyma12g33280 and Glyma19g28070) in the network were among the predicted candidate genes. These hub genes were confirmed by qRTPCR with seven extreme genotypes, thus, five high HSW genotypes (P048, P130, P227, P589 and P602) and two low HSW genotypes (P415 and P579) in seed sampled at R5 and R7 stages. With the exception of Glyma12g33280, the remaining three hub genes (Glyma06g44510, Glyma08g06420 and Glyma19g28070) were relatively expressed higher in the high HSW genotypes at R5 stage compared with low HSW genotypes (Fig. 6). However, the expression were not consistent in the R7 stage.

\section{Discussion}

\section{Phenotype variation and genetic basis of seed weight in YHSBLP}

Identification of molecular markers associated with a trait of interest is one of the prerequisites of molecular breeding. Soybean seed weight is one of the most critical traits having direct effect on yield as a yield component, 

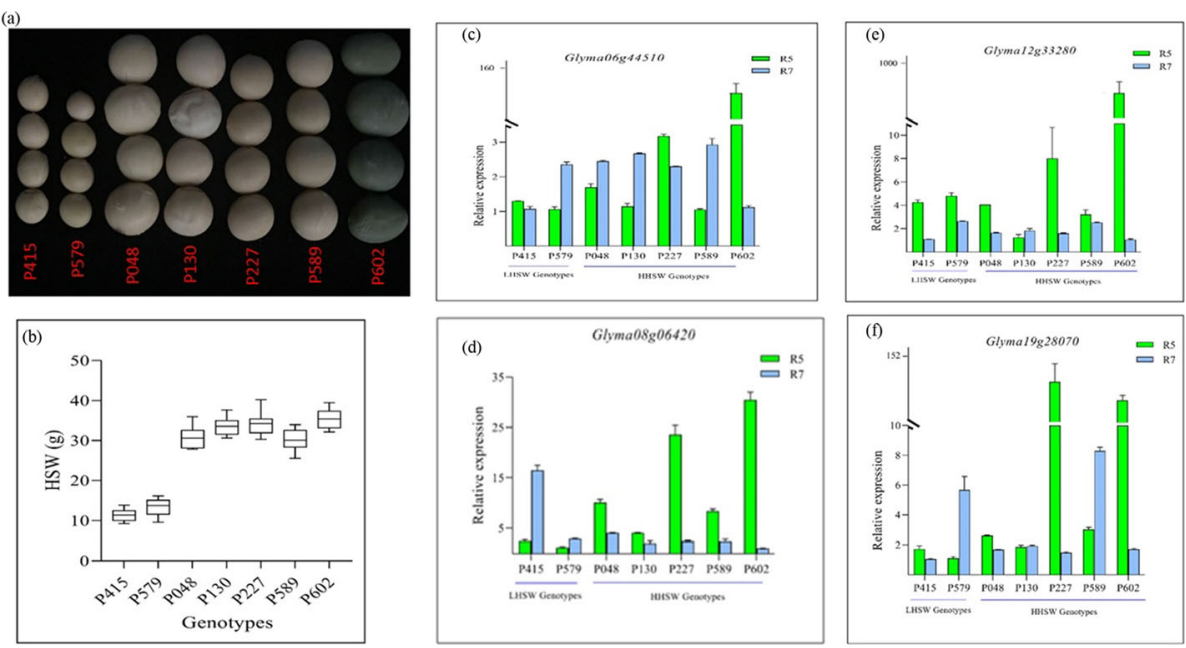

Fig. 6 Seed weight among selected genotypes and relative expression of 4-hub genes by qRT-PCR. a \& $\mathbf{b}$ Phenotypic characterization of selected genotypes for qRT-PCR. Relative expression of Glyma06g44510 (c), Glyma08g06420 (d), Glyma12g33280 (e) and Glyma19g28070 (f) by qRT-PCR with seed at R5 and R7 stages of seed development. (LHSW and HHSW represent Low HSW and High HSW, respectively. The error bars represent standard error of means)

with much importance as a quality attribute and its influence on seed use [7-9]. The seed weight (HSW) ultimately determines the economic return on soybean production. However, this trait is a complex trait controlled by many polygenes with both major and minor effects, which is also significantly influenced by the environment and genotype by environment interaction. This makes screening on the basis of phenotype alone very difficult and inefficient. Hence, identifying QTL/QTNs for marker-assisted breeding for seed weight would be beneficial.

The HSW in soybean is a typical quantitative trait which is easily influenced by genotype, environment or by genotype $\times$ environment interaction $(\mathrm{G} \times \mathrm{E})$ which are in consonance with several earlier studies [30,46]. There was much variability in the YHSBLP (7.24-39.70g) (Additional File 1: Table S1) which together with larger population size and high SNPs enhanced effectiveness and efficiency of QTN detection via SNP-trait association [49, 50, 59]. The HSW showed normal distribution in each environment (E1, E2, E3 and E4) (Fig. 1) coupled with high $h^{2}$ which indicated that variation in HSW is controlled by multiple genetic loci with both major and minor effects (Table 1). This study utilized 573 recently developed breeding line population (YHSBLP) compared with most of the earlier studies that used wild accession, landraces and elite cultivars $[24,26,27,30,46]$. The high genetic variability shows the potentials of YHSBLP for genetic improvement aimed at seed weight [60].

\section{QTNs detected by the single and multi-locus models and their comparisons}

Most of the earlier reported QTL/QTNs were performed with $<500$ accessions and SNPs were $<60,000[29,30$, 46-48]. The power of detection in GWAS is constrained by the population size, genetic diversity as well as genome coverage/number of SNPs, linkage disequilibrium, and statistical models $[17,49,50]$. Therefore, this study used two SLMs (MLM \& CMLM) together with six MLMs (mrMLM, FASTmrMLM, FASTmrEMMA, pLARmEB, pKWmEB and ISIS EM-BLASSO) to identify genomic regions associated with seed weight. A total of 39 SNPs were significantly associated with HSW by the two single-locus models (MLM \& CMLM) across ten out of the 20 chromosomes viz. Chr.01, Chr.02, Chr.04, Chr.08, Chr.09, Chr.11, Chr.13, Chr.14, Chr.15, Chr.16 and Chr.17 (Additional File 2: Table S2; Additional File 3: Fig. S1; Additional File 4: Fig. S1). Out of these, 24 were mutually detected by both MLM \& CMLM whereas 6 and 9 SNPs were exclusively detected by CMLM and MLM, respectively (Additional File 5: Fig. S3A). On the other hand, two hundred and nine SNPs were associated with HSW via the six MLMs across the 20 chromosomes (Additional File 2: Table S2). Each of the six models detected varied number of SNPs: pLARmEB (82) > mrMLM (75) > pKWmEB (61) > FASTmrMLM (56) > ISIS EM-BLASSO (47) > FASTmrEMMA (32). This indicates varied detection of each model. Also, pairwise comparisons of the 6 models demonstrated that each of the models has the power to detect SNPs concurrently from each other, though no SNP was detected by all the six models simultaneously. For the example mrMLM \& FASTmrMLM concurrently detected 44 SNPs followed by mrMLM \& pKWmEB (22) and least by ISIS EM-BLASSO with FASTmrMLM (11) and FASTmrEMMA (11) (Additional File 2: Table S3). Multiple multi-site association analysis methods cannot only improve the reliability of QTNs detected, but also complement each other to detect more QTNs. In this study, two important QTNs: $q H S W-$ 
8-1 and $q H S W-19-4 / q H S W-19-5$ harbored 2 of the 4 hub genes identified were solely detected by only the MLMs. This also buttresses the usefulness of MLMs in GWAS

In comparison of the two SLMs and six MLMs, 17 common SNPs were detected on 6 chromosomes (Chr.02, Chr.08, Chr.09, Chr.11, Chr.13 and Chr.14) (Additional File 5: Fig. S3B; Additional File 4: Fig. S2). Even though, the threshold of the significance in SLMs was adjusted to 4 instead of 4.79 (Bonferroni correction), the number of significant SNPs detected by SLMs were lower than those detected by MLMs, which confirm the robustness and power of detection of the later models. The combination of both SLMs and MLMs enhanced the detection of both major and minor QTNs. Several SNPs were detected in specific environment which are in consonance with ANOVA, indicating that seed weight of Chinese summer sowing soybean is also regulated by the environment. The combination of the two SLMs as well as the six MLMs complemented each other in identifying 231 QTNs which could have been lost in either of the models. However, the MLMs proved to be more robust and powerful in detecting more SNPs than the SLMs. Similar trend have been reported in soybean [34], cotton [35], maize [61] and flax [62] where more number of significant SNPs were detected by the MLMs comparing with SLMs. Therefore, the use of multi-locus models with their power of detection can facilitate genomic selection in breeding.

The stability of QTL/QTNs is essential for the use in a breeding program. Ninety-four of the QTNs identified in this study co-localized with several earlier reported QTL/QTNs could be exploited and integrated into breeding (Additional File 2: Table S2). Whereas 137 novel QTNs are being reported for the first time based on QTL/QTNs documented on SoyBase as some recently published reports need further verification. This could be attributed to the diverse background of our recently developed breeding lines pointing to their potential for breeding programs. Out of 137 novel loci, 22 were detected in at least two environments by at least two models (Additional File 2: Table S2). Also, allele effect on seed weight by haplotype block analyses could be utilized to conduct haplotype-based breeding scheme to develop genotypes with desirable seed weight whilst exploring the vast genetic base of YHSBLP.

\section{Candidate genes predicted and further analysis}

Identification and utilization of candidate genes is one of the key objectives of GWAS. So far few genes have been validated and confirmed to regulate seed weight/size in soybean [63-65] compared with Arabidopsis and rice [66-71]. A mature seed consists of embryo, endosperm and seed coat derived from zygote, fertilized central cell and maternal integuments, respectively [67, 72]. Seed weight/size is also dependent on cell size and its proliferation, flower development, sucrose transport and other related activities which are regulated by several signaling pathways [66-68, 73-75]. Sucrose which is the major sugar composition accounts $97 \%$ in embryo during seed development process [76]. In our study, five of the thirtysix candidate genes are related with sucrose transport (Additional File 6: Table S4). For example, within the haplotype block Gm06_13796257-13926598 had a significant SNP at 13909376 bp ( $q H S W-6-5)$ with three sucrose transporter genes (Glyma06g17520, Glyma06g17530 and Glyma06g17540) at $40.04 \mathrm{~kb}, 21.72 \mathrm{~kb}$ and $7.62 \mathrm{~kb}$ upstream of the SNP, respectively (Additional File 6: Table S4). These genes are 99.68, 99.62 and $99.61 \%$ similar to AtSWEET12, AtSWEET13 and AtSWEET10, respectively. Again, another sucrose transporter gene Glyma11g27070 which is ortholog to AtSWEET15 was found $450.05 \mathrm{~kb}$ downstream of the SNP at 27075467 (Gm11_2686106427075467). Less is known of the role of GmSWEET genes in seed development and its related traits in soybean $[20$, 77] compared to other crops [78, 79].

The functional network obtained from SoyNet revealed 4 hub-genes viz. Glyma06g44510, Glyma08g06420, Glyma12g33280 and Glyma19g28070 in our predicted candidate genes (Additional File 7: Fig. S4). Although, the functional relationship depicted in the co-expression network did not include all the 36 candidate genes predicted in this study, it gives better clues about 15 genes out of the predicted genes together with other genes which were not captured in this study. There is intensive literature on the interaction of ribosomal proteins and kinesin proteins in regulating embryo/seed size and radicle growth [80]. Such interaction was evident in the network constructed in this study. Some members of kinesin protein have been demonstrated to regulate embryo/seed size in rice [81, 82]. The network captured two genes (Glyma17g17850 and Glyma17g18360) within the block Gm17_1517812315400615 (SNP at 15346512). These two genes belong to Subtilase family protein and CYCLIN D3;2 which are involved in seed coat and other essential processes in seed weight regulation, respectively [73, 83]. Similarly, Glyma10g38580 which is a K-box region and MADS-box transcription factor was captured in the network. MADSbox genes have been reported to be jack of all traits [84]. The network also covered the two genes from multidrug resistance-associated protein 6; $\mathrm{ABC}$ transporter transmembrane region; $A B C$ transporter proteins which have recently demonstrated to enhance seed yield and quality in chickpea [51]. The functional co-expression network capturing some key genes which had some orthologs demonstrated to regulate seed development, implies that integration of co-expression can be one of the strategies to identify keys underlying major agronomic traits in crops. 
Cis-acting regulatory elements (CAREs) are major switches for transcriptional regulation of a dynamic network of gene expression regulating different biological processes such as abiotic stress responses, hormone responses and developmental processes [85]. Further bioinformatics analysis showed that three of the four hub genes (Glyma06g44510, Glyma12g33280, Glyma19g28070) together with others possess seed-related cis-elements, thus, GCN4_motif, MSA-like, RY-element, circadian clock and ABRE element (Additional File 6: Table S6) $[53-55,86]$. The predicted candidate genes especially the hub-genes will be validated in our future works to ascertain their actual roles in seed weight regulation via overexpression, CRISPR/Cas9 and other functional methods.

\section{Conclusion}

The genetic architecture of YHSBLP planted in summer are regulated by varied QTNs unevenly distributed on the 20 chromosomes. Seventeen QTNs were detected concurrently representing 43.54 and $8.13 \%$ of the total QTNs by the two SLMs and six MLMs, respectively. Among the six MLMs, mrMLM and pLARmEB are most robust in detecting more QTNs. A number of SNPs including Gm08_15803242, Gm09_3461722, Gm13_ 43480280 and Gm14_40721910/ Gm14_40721920 detected by multiple models in at least two environments could further be validated and used for marker-assisted breeding (MAB). In all, thirty-six candidate genes that may regulate seed weight in soybean were identified. These will be useful in comparative genomics aimed at unraveling the molecular mechanism underlying seed development/weight in soybean. Four hub genes viz., Glyma06g44510, Glyma08g06420, Glyma12g33280 and Glyma19g28070, were identified by the integration of co-expression network. All the hub genes were found to have higher expression in the seeds of high HSW genotypes than low HSW at R5 stage except Glyma12g33280, therefore, they could be cloned to study their regulating role in seed development. The findings in this study would be valuable for breeding geared toward desirable seed weight via MAB and haplotype-based breeding scheme.

\section{Materials and methods}

\section{Germplasm, field evaluation and phenotyping}

The tested panel named as YHSBLP includes a total of 573 breeding lines adapted to Chinese Yangtze-Huai river region for both grain and vegetable soybean use. All lines were obtained from National Center for Soybean Improvement, Nanjing Agricultural University (NAU), Nanjing-China. This population was mainly derived from the core parents in breeding programs (Nannong 86-4, Nannong 88-48, Yuchu 4 and Nannongcaidou 5), other local and foreign elite cultivars. The hybrid method was used to select pods in $\mathrm{F}_{2}-\mathrm{F}_{4}$ generation of each combination, and the high-yield and good plant type were selected in $\mathrm{F}_{5}-\mathrm{F}_{6}$ generation. The lines selected for this study comprised high-yield and stable lines in $\mathrm{F}_{8}-\mathrm{F}_{14}$ generation.

Field evaluation of the population was conducted in Jiangpu (Latitude $33^{\circ} 03^{\prime} \mathrm{N}$; Longitude $118^{\circ} 63^{\prime} \mathrm{E}$ ), Experimental Station of NAU in summer 2013, 2014, 2017 and 2018 coded E1, E2, E3 and E4, respectively (Additional File 9: Table S8, or available in the National Center for Soybean Improvement website, http://ncsi.njau. edu.cn/info/1150/2069.htm). The lines were planted in randomized complete block design with $50 \mathrm{~cm} \times 50 \mathrm{~cm}$ hill plots in 3 replications. All recommended agronomic and cultural practices were followed. A 100-seed weight (HSW) for each replication was measured with 2 technical repeats at $13 \%$ moisture content via electronic balance. The mean of each genotype from the 2 technical repeats were computed for each replication.

\section{Statistical analysis of 100 -seed weight}

Data collected were subjected to analysis of variance (ANOVA) in SAS (SAS Institute, 2010. SAS/STAT software version 9.2. SAS Institute Inc., Cary, NC) following statistical model:

$$
y_{m l o}=\mu+G_{m}+E_{l}+G E_{m l}+R_{o(l)}+\varepsilon_{m l o},
$$

where $y_{m l o}$ stands for the individual observation of $m l^{t h}$ experiment unit, $\mu$ is the total average HSW, $G_{m}$ is the effect of the $m^{\text {th }}$ genotype, $E_{l}$ is the effect of the $l^{t h}$ environment, $G E_{m l}$ is the interaction effect between the $m^{\text {th }}$ genotype and the $l^{\text {th }}$ environment, $R_{o(l)}$ is the effect of the $o^{\text {th }}$ block within the $l^{\text {th }}$ environment, and $\varepsilon_{m l o}$ is the residual error. All factors were considered as random.

Descriptive statistics such as mean, standard error of mean, kurtosis and skewness were computed in each environment with OriginPro 8 Statistical Software (Origin Corporation, Northampton, MA, USA) whereas variation in HSW among the genotypes was visualized using Violin plot with ggplot2 package in R [87]. Broad-sense heritability $\left(h^{2}\right)$ were computed for the combined environment following $h^{2}=\sigma_{g}^{2} /\left(\sigma_{g}^{2}+\sigma_{g e}^{2} / n+\sigma_{e}^{2} / n r\right)$ where $\sigma_{g}^{2}$ is the genotypic variance, $\sigma_{g e}^{2}$ is the genotype by environment interaction variance, $\sigma_{e}^{2}$ is the error variance, $n$ is the number of environments, and $r$ is the number of replications [88].

\section{Genotyping}

The DNA sample of each accession was genotyped by the Restriction site-associated DNA sequencing (RADseq) technology to generate high throughput SNPs. Briefly, the genomic DNA of the 573 accessions was extracted from young leaves using the CTAB method [89]. All DNA fragments between $400 \mathrm{bp}$ and $600 \mathrm{bp}$ were 
obtained by TaqI digestion. The fragments were sequenced using an Illumina HiSeq 2000 instrument with a paired-end reads length of $90 \mathrm{bp}$ (including $6 \mathrm{bp}$ index) of read length [90]. All sequence reads were aligned against the reference Glyma.Wm82.a1.v1.1 [91] using SOAP2 software [92], and SNP calling was performed by RealSFS software [93]. The criteria filtering SNPs of the 573 accessions were as follows: a rate of missing and heterozygous allele calls $\leq 30 \%$, minor allele frequency (MAF) $\geq 5 \%$. The fastPHASE software [94] was used for genotyping the SNP imputation after the heterozygous alleles were turned into missing alleles, resulting in 61, 166 high-quality SNP markers (Available on NCBI database: PRJNA648781, or available in the National Center for Soybean Improvement website, http://ncsi.njau.edu. cn/info/1149/2070.htm). The SNP density plot was constructed with CMPlot package in R [95].

\section{Genetic diversity, population structure and haplotype block}

The filtered SNPs were further pruned using the indep-pairwise command option of pLINK. The pruned SNPs were then used to estimate population structure via ADMIXTURE V1.3.0 software [96]. In the ADMIXTURE setting, the number of clusters (K) was set from 1 to 10 initially; then, each $\mathrm{Q}$ and the relevant $P$-value was estimated. The most likely number of subpopulations was determined using the method described in Evanno et al. [97]. Principal Component Analysis (PCA) was carried out in Trait Analysis by aSSociation, Evolution and Linkage (TASSEL) software, version 5.2 [98]. A pairwise Nei's genetic distance matrix was calculated in TASSEL for Neighbor-joining tree construction.

\section{Association mapping and haplotype block analysis}

Two SLMs of GWAS viz. MLM $(\mathrm{Q}+\mathrm{K})$ and compression MLM $(\mathrm{CMLM})(\mathrm{PCA}+\mathrm{K})$ were conducted in TASS EL 5.2 [98] and Genome wide Association Prediction Tool (GAPIT) environment in $\mathrm{R}$ [99], respectively, where $\mathrm{Q}$ matrix was obtained from population structure computed in ADMIXTURE V1.3.0 software [96], kinship matrix (K) was estimated in each software and 3 PCs were used for PCA in the CMLM. A threshold value $\left(-\log _{10}(P) \geq 4.00\right)$ was adopted to declare a significant association of SNPs with seed weight.

Six MLMs viz. mrMLM [100], FASTmrMLM [101], FASTEMMA [31], pLARmEB [102], pKWmEB [103] and ISIS EM-BLASSO [104] were computed in R with the package mrMLM.GUI (https://cran.r-project.org/ web/packages/mrMLM.GUI/index.html). In these models, Q matrix was used to account for population structure whilst the kinship matrix $(\mathrm{K})$ was computed in the mrMLM.GUI environment. A critical LOD value was set at 3 . The SNPs detected by at least 2 models in least one environment was considered as relatively stable SPNs. QTL naming was done following the nomenclature of McCouch et al. [105], thus starting with ' $q$ ', followed by an abbreviation of the trait name (HSW, hundred seed weight) and the name of the chromosome, followed by the number of QTL detected on the same chromosome.

Haplotype block analysis of the relatively stable SNPs across the 2 of the single-locus plus at least 2 multilocus models was conducted in Haploview software with the four-gamete rule method with default parameters in Haploview software version 4.2 [106, 107]. Duncan Range Multiple test (pairwise comparison) was used to assess variation in seed weight among accession groupings in each haplotype block at the significant level of $P \leq 0.05$.

\section{Candidate genes prediction and analysis}

Potential candidate genes were retrieved within $500 \mathrm{~kb}$ of significant SNPs detected in at least 2 environments by either single-locus models or multi-locus models in the $G$. max William 82 reference gene models 1.0 in SoyBase [108]. The functional annotations of model genes downloaded from SoyBase which were screened manually. The predicted candidate genes were further compared with their orthologs in other legume crops to confirm their functions in relation to seed development using an Integrative Platform to study gene function and genome evolution in legumes(LegumePI) version 2 (http://plantgrn. noble.org/LegumeIP) [109] and Legume Information System (LIS) (https://legumeinfo.org/) [110].

The sequence of $1.5 \mathrm{~kb}$ upstream (before ATG) of each gene was obtained from Phytozome database (https:// phytozome.jgi.doe.gov). The obtained sequences were then submitted to PlantCare database available on http://bioinformatics.psb.ugent.be/webtools/plantcare/ html to identify cis-elements related to seed-related functions in the promoter region of each gene [111].

A functional network of protein-protein interaction among the predicted candidate genes and other related genes were obtained via SoyNet (https://www.inebio.org/ soynet/serach.php) [58]. The functional network derived from SoyNet was then visualized in standalone version of Cytoscape software [112] and NetworkAnalyst 3 [113].

\section{Validation of hub genes by real time quantitative polymerase chain reaction (qRT-PCR)}

Seven genotypes of YHSBLP with extreme differences in HSW (comprising 2 and 5 genotypes with low HSW and high HSW, respectively) were sampled at R5 and R7 stages of seed development [114] of 2019 summer season at Jiangpu Experimental station. Total RNA was isolated using Plant RNA Extract Kit (TIANGEN Co., Ltd. China) and complementary DNA (cDNA) synthesis obtained by 
using HiScript II QRT SuperMix for qPCR (+gDNA wiper) (Vazyme Biotech, Nanjing, China). The enzyme 2x ChamQ $^{\text {in }}$ SYBR qPCR Master Mix Kit (Vazyme Biotech, Nanjing, China) was used following standard protocol and program in a Light Cycler 480 system (Roche, Roche Diagnostic, Basel, Switzerland). Three biological and three technical replicates were used. The GmActin 11 (Glyma18g52780) was used as a housekeeping gene to normalize the relative expression level in R5 and R7 stages of the selected genotypes seed. The primers used for qRTPCR are presented in Additional File 8: Table S7.

\section{Supplementary information}

Supplementary information accompanies this paper at https://doi.org/10 1186/s12870-020-02604-z.

Additional file 1 : Table S1. Descriptive statistics of SW in YHSBLP evaluated in 4 environments.

Additional file 2 : Table S2. Quantitative trait nucleotides significantly associated with hundred seed weight identified by the single-locus models and multi-locus models. Table S3. Pairwise comparison of the 8 GWAS models.

Additional file 3 : Fig. S1. Manhattan plots (left) and QQ-plots (right) for GWAS of the 573 accessions for HSW in E1 (A), E2 (B), E3 (C) and E4 (D) using CMLM (PCA + K). The threshold of 4 was adopted with a blue line in the Manhattan plots. The $X$-axis represents chromosome number and $Y$-axis represents $-\log _{10}(P)$. The $X$ and $Y$ axis in the QQ plots represent the expected and observed $-\log _{10}(P)$, respectively. Red line in the QQ-plots with the shaded regions indicate a 95\% confidence interval.

Additional file 4 : Fig. S2. Manhattan plots (left) and QQ-plots (right for GWAS of the 573 accessions for HSW in E1 (A), E2 (B), E3 (C) and E4 (D) using MLM $(Q+K)$. The threshold of 4 was adopted with a blue line in the Manhattan plots. The $X$-axis represents chromosome number and $Y$ axis represents $-\log _{10}(P)$. The $X$ and $Y$ axis in the QQ plots represent the expected and observed $-\log _{10}(P)$, respectively. Red line in the QQ-plots with the shaded regions indicate a 95\% confidence interval.

Additional file 5 : Fig. S3. Number of common significant SNPS detected between models. (A). A number of common SNPs detected by the two single-locus models (MLM-blue color \& CMLM-brown color). (B). A number of common SNPs detected by the 2 single-locus models (MLM \& CMLM-blue color) and six multi-locus models (mrMLM, FASTmrMLM, FASTEMMA, pLARmEB, pKWmEB and ISIS EM-BLASSO-brown color).

Additional file $\mathbf{6}$ : Table S4. Potential candidate genes underlying stable SNPs. Table S5. Candidate genes expression across the various tissues and seed developmental stages obtained from SoyBase. Table S6. Cis-acting regulating elements related to seed development obtained from PlantCARE.

Additional file 7 : Fig. S4. Functional gene network of candidate genes predicted in this study and other related genes obtained from SoyNet. (A). Dense-interaction network obtained from standlone version of Cytoscape software. (B). The 4-hub genes and other visualized in NetworkAnalyst version 3 . The node colors represent between degrees of interaction: red, pink, purple and blue represent very high, high, moderate and low levels of interaction, respectively.

Additional file 8 : Table S7. Primers used for QRT-PCR.

Additional file $\mathbf{9}$ : Table S8. Phenotypic data (average) of 573 accessions used in this study.

\section{Abbreviations}

CAREs: Cis-acting regulatory elements; GWAS: Genome-wide association study; MLMs: Multi-locus models; QTL: Quantitative trait loci;

QTNs: Quantitative trait nucleotides; RAD-seq: Restriction site-associated DNA sequencing; SLMs: Single-locus models; SNPs: Single nucleotide polymorphisms

\section{Acknowledgements}

The authors are thankful to all the team- and lab mates and friends who supported us during the research project and writing of the manuscript.

\section{Authors' contributions}

T.J.Z. conceived and designed the experiments. B.K., Z.L.W., Y.L.Z. and W.L.Y performed the experiments. B.K. \& J.Y.F analyzed the data and drafted the manuscript. T.J.Z. \& J.Y.F revised the paper. The author(s) read and approved the final manuscript.

\section{Funding}

This work was supported by the National Key R \& D Program of China (2016YFD0100304, 2018YFD0100800), the National Natural Science Foundation of China (31871646), the MOE Program for Changjiang Scholars and Innovative Research Team in University (PCSIRT_17R55), the Fundamental Research Funds for the Central Universities (KYT201801), the Jiangsu Collaborative Innovation Center for Modern Crop Production (JCICMCP) Program.

\section{Availability of data and materials}

With the exception of SNP datasets, all data generated or analyzed during this study are included in this published article and its supplementary information files. The SNP dataset used in the current study are available in the Sequence Read Archive (SRA) at NCBI (SRA accession: PRJNA648781) repository and on the website of the National Center for Soybean Improvement, http://ncsi.njau.edu.cn/zygx.htm.

Ethics approval and consent to participate

Not applicable.

Consent for publication

Not applicable.

\section{Competing interests}

The authors declare no conflict of interest.

Received: 7 April 2020 Accepted: 16 August 2020

Published online: 01 September 2020

\section{References}

1. Hickey LT, Hafeez AN, Robinson H, Jackson SA, Leal-Bertioli SC, Tester M, Gao C, Godwin ID, Hayes BJ, Wulff BB. Breeding crops to feed 10 billion. Nat Biotechnol. 2019:37:744-54

2. Dita MA, Rispail N, Prats E, Rubiales D, Singh KB. Biotechnology approaches to overcome biotic and abiotic stress constraints in legumes. Euphytica. 2006;147(1-2):1-24.

3. Pandey MK, Roorkiwal M, Singh VK, Ramalingam A, Kudapa H, Thudi M, Chitikineni A, Rathore A, Varshney RK. Emerging genomic tools for legume breeding: current status and future prospects. Front Plant Sci. 2016;7:455.

4. Sparvoli F, Bollini R. Cominelli E. In: DeRon AM, editor. Grain Legumes. Berlin: Springer; 2015. p. 291-318.

5. Messina M. Soy and health update: evaluation of the clinical and epidemiologic literature. Nutrients. 2016;8(12):754.

6. Messina MJ. Legumes and soybeans: overview of their nutritional profiles and health effects. Am J Clin Nutri. 1999;70(3):439-50.

7. Cui Z, James A, Miyazaki S, Wilson RF, Carter TE Jr. Breeding specialty soybeans for traditional and new soyfoods. In: Liu K, editor. Soybeans as functional foods and ingredients: AOCS Press; 2004. p. 74-332.

8. Gandhi A. Quality of soybean and its food products. Inter Food Res J. 2009;16(1):119.

9. Li N, Xu R, Li Y. Molecular networks of seed size control in plants. Annual Rev Plant Biol. 2019;70:1-30.

10. Liang $\mathrm{H}$, Lanjie $\mathrm{XU}$, Yongliang $\mathrm{YU}$, Yang $\mathrm{H}$, Wei $\mathrm{D}$, Zhang $\mathrm{H}$. Identification of QTLS with main, epistatic and QTL by environment interaction effects for seed shape and hundred-seed weight in soybean across multiple years. J Genet. 2016;95(2):475. 
11. Teng W, Feng L, Li W, Wu D, Zhao X, Han Y, Li W. Dissection of the genetic architecture for soybean seed weight across multiple environments. Crop Pasture Sci. 2017;68(4):358-65

12. Wu D, Zhan Y, Sun Q, Xu L, Lian M, Zhao X, Han Y, Li W. Identification of quantitative trait loci underlying soybean (Glycine max [L.] Merr.) seed weight including main, epistatic and QTL $\times$ environment effects in different regions of Northeast China. Plant Breed. 2018;137(2):194-202.

13. Wang J, Chu S, Zhang H, Zhu Y, Cheng H, Yu D. Development and application of a novel genome-wide SNP array reveals domestication history in soybean. Sci Rep. 2016;6:20728.

14. Han Y, Zhao X, Liu D, Li Y, Lightfoot DA, Yang Z, Zhao L, Zhou G, Wang Z, Huang $L$. Domestication footprints anchor genomic regions of agronomic importance in soybeans. New Phytol. 2016:209(2):871-84.

15. Lee G-A, Crawford GW, Li L, Yuka S, Xuexiang C. Archaeological soybean (Glycine max) in East Asia: does size matter? PLoS One. 2011;6(11):e26720.

16. Zhou Z, Yu J, Zheng W, Zhiheng G, Jun L, Weiyu L, Yanjun Y, Liping S, Yingjun Z, Yanming M. Resequencing 302 wild and cultivated accessions identifies genes related to domestication and improvement in soybean. Nat Biotechnol. 2015:33(4):408-14.

17. Gupta PK, Rustgi S, Kulwal PL. Linkage disequilibrium and association studies in higher plants: present status and future prospects. Plant Mole Biol. 2005;57(4):461-85.

18. Rafalski A. Applications of single nucleotide polymorphisms in crop genetics. Curr Opin Plant Biol. 2002;5(2):94-100.

19. Asíns MJ. Present and future of quantitative trait locus analysis in plant breeding. Plant Breed. 2002;121(4):281-91.

20. Miao L, Yang S, Zhang K, He J, Wu C, Ren Y, Gai J, Li Y. Natural variation and selection in GmSWEET39 affect soybean seed oil content. New Phytol. 2019; 225(4):1651-66.

21. Zhang H, Hao D, Sitoe HM, Yin Z, Hu Z, Zhang G, Yu D. Genetic dissection of the relationship between plant architecture and yield component traits in soybean (Glycine max) by association analysis across multiple environments. Plant Breed. 2015;134(5):564-72.

22. Zhang J, Song Q, Cregan PB, Jiang G-L. Genome-wide association study, genomic prediction and marker-assisted selection for seed weight in soybean (Glycine max). Theor Appl Genet. 2016;129(1):117-30.

23. Fang C, Ma Y, Wu S, Liu Z, Wang Z, Yang R, Hu G, Zhou Z, Yu H, Zhang M. Genome-wide association studies dissect the genetic networks underlying agronomical traits in soybean. Genome Biol. 2017;18(1):161.

24. Copley TR, Duceppe MO, O'Donoughue LS. Identification of novel loci associated with maturity and yield traits in early maturity soybean plant introduction lines. BMC Genomics. 2018;19(1):167.

25. Li X, Zhang X, Zhu L, Bu Y, Wang X, Zhang X, Zhou Y, Wang X, Guo N, Qiu L. Genome- wide association study of four yield-related traits at the R6 stage in soybean. BMC Genet. 2019;20(1):39.

26. Chaudhary J, Patil GB, Sonah H, Deshmukh RK, Vuong TD, Valliyodan B, Nguyen HT. Expanding Omics Resources for Improvement of Soybean Seed Composition Traits. Front Plant Sci. 2015;6(31)

27. Zhao X, Dong H, Chang H, Zhao J, Teng W, Qiu L, Li W, Han Y. Genome wide association mapping and candidate gene analysis for hundred seed weight in soybean [Glycine max (L.) Merrill]. BMC Genom. 2019;20(1):648.

28. Sun YN, Pan JB, Shi XL, Du XY, Wu Q, Qi ZM, Jiang HW, Xin DW, Liu CY, Hu $\mathrm{GH}$. Multi-environment mapping and meta-analysis of 100-seed weight in soybean. Mole Biol Rep. 2012;39(10):9435-43.

29. Assefa T, Otyama PI, Brown AV, Kalberer SR, Kulkarni RS, Cannon SB. Genomewide associations and epistatic interactions for internode number, plant height, seed weight and seed yield in soybean. BMC Genomics. 2019;20(1):52.

30. Yan L, Hofmann N, Li S, Ferreira ME, Song B, Jiang G, Ren S, Quigley C, Fickus E, Cregan P. Identification of QTL with large effect on seed weight in a selective population of soybean with genome-wide association and fixation index analyses. BMC Genomics. 2017;18(1):529.

31. Wen Y-J, Zhang H, Ni Y-L, Huang B, Zhang J, Feng J-Y, Wang S-B, Dunwell JM, Zhang Y-M, Wu R. Methodological implementation of mixed linear models in multi-locus genome-wide association studies. Brief Bioinfor. 2017; 19(4):700-12.

32. Zhang Y-M, Jia Z, Dunwell JM. The applications of new multi-locus GWAS methodologies in the genetic dissection of complex traits. Front Plant Sci. 2019;10:100

33. Chang M, He L, Cai L. An Overview of Genome-Wide Association Studies. In: Huang T, editor. Computational Systems Biology: Methods and Protocols. New York: Springer New York; 2018. p. 97-108.8.
34. Chang F, Guo C, Sun F, Zhang J, Wang Z, Kong J, He Q, Sharmin RA, Zhao T. Genome-wide association studies for dynamic plant height and number of nodes on the Main stem in summer sowing soybeans. Front Plant Sci. 2018:9:1184.

35. Li C, Yuanzhi F, Runrun S, Yuanyuan W, Qinglian W. Single-locus and multilocus genome-wide association studies in the genetic dissection of Fiber quality traits in upland cotton (Gossypium hirsutum L.). Front Plant Sci. 2018; 9:1083.

36. Zhang K, Liu S, Li W, Liu S, Li X, Fang Y, Zhang J, Wang Y, Xu S, Zhang J, Song J. Identification of QTNs controlling seed protein content in soybean using multi-locus genome-wide association studies. Front Plant Sci. 2018;9: 1690

37. Zhang C, Li L, Liu Q, Gu L, Huang J, Wei H, Wang H, Yu S. Identification of loci and candidate genes responsible for fiber length in upland cotton (Gossypium hirsutum L.) via association mapping and linkage analyses. Front. Plant Sci. 2019;10:53

38. Schaefer R, Michno J-M, Jeffers J, Hoekenga OA, Dilkes BP, Baxter IR, Myers C. Integrating co-expression networks with GWAS to prioritize causal genes in maize. Plant Cell. 2018:30:2922-42.

39. Sarkar NK, Kim Y-K, Grover A. Coexpression network analysis associated with call of rice seedlings for encountering heat stress. Plant Mole Biol. 2014; 84(1-2):125-43

40. Zhang H, Wang ML, Schaefer R, Dang P, Jiang T, Chen C. GWAS and coexpression network reveal Ionomic variation in cultivated Peanut. J Agric Food Chem. 2019;67(43):12026-36.

41. Angelovici R, Batushansky A, Deason N, Gonzalez-Jorge S, Gore MA, Fait A, DellaPenna D. Network-guided GWAS improves identification of genes affecting free amino acids. Plant Physiol. 2017;173(1):872-86.

42. Yang S, Miao L, He J, Zhang K, Li Y, Gai J. Dynamic Transcriptome changes related to oil accumulation in developing soybean seeds. Inter J Mole Sci. 2019;20(9):2202

43. Wu Z, Wang M, Yang S, Chen S, Chang X. A global coexpression network of soybean genes gives insight into the evolution of nodulation in nonlegumes and legumes. New Phytol. 2019;223:2104-19.

44. Palumbo MC, Zenoni S, Fasoli M, Massonnet M, Farina L, Castiglione F, Pezzotti M, Paci P. Integrated network analysis identifies fight-Club nodes as a class of hubs encompassing key putative switch genes that induce major Transcriptome reprogramming during grapevine development. Plant Cell. 2014;26(12):4617-35

45. Das S, Meher PK, Rai A, Bhar LM, Mandal BN. Statistical Approaches for Gene Selection, Hub Gene Identification and Module Interaction in Gene CoExpression Network Analysis: An Application to Aluminum Stress in Soybean (Glycine max L.). PLoS One. 2017;12(1):e0169605.

46. Li J, Zhao J, Li Y, Gao Y, Hua S, Nadeem M, Sun G, Zhang W, Hou J, Wang X Identification of a novel seed size associated locus SW9-1 in soybean. Crop J. 2019;7(4):548-59.

47. Xue Z, Wenjing L, Xiaoyue Z, Jinyang W, Zhiyang L, Han Y, Li W. Genome wide association mapping and candidate gene analysis for seed shape in soybean [Glycine max (L.) Merri]. Crop Pasture Sci. 2019:70(8):684-93.

48. Contreras-Soto RI, Mora F, Oliveira MARD, Higashi W, Scapim CA, Schuster I. A genome-wide association study for agronomic traits in soybean using SNP markers and SNP-based haplotype analysis. PLoS One. 2017;12(2).

49. Josephs EB, Stinchcombe JR, Wright SI. What can genome-wide association studies tell us about the evolutionary forces maintaining genetic variation for quantitative traits? New Phytol. 2017;214(1):21-33.

50. Xu Y, Liu X, Fu J, Wang H, Wang J, Huang C, Prasanna BM, Olsen MS, Wang $G$, Zhang A. Enhancing genetic gain through genomic selection: from livestock to plants. Plant Comm. 2019;1:100005.

51. Basu U, Upadhyaya HD, Srivastava R, Daware A, Malik N, Sharma A, Bajaj D, Narnoliya L, Thakro V, Kujur A. ABC transporter-mediated transport of glutathione conjugates enhances seed yield and quality in chickpea. Plant Physiol. 2019;180(1):253-75.

52. Severin AJ, Woody JL, Bolon YT, Joseph B, Diers BW, Farmer AD, Muehlbauer GJ, Nelson RT, Grant D, Specht JE. RNA-Seq atlas of Glycine max: a guide to the soybean transcriptome. BMC Plant Biol. 2010;10(1):160.

53. Onodera Y, Suzuki A, Cy WH, Takaiwa F. A rice functional transcriptional activator, RISBZ1, responsible for endosperm-specific expression of storage protein genes through GCN4 motif. J Biol Chem. 2001;276(17):14139-52.

54. Ito M, Iwase M, Kodama H, Lavisse P, Komamine A, Nishihama R, Machida Y, Watanabe A. A novel cis-acting element in promoters of plant B-type cyclin genes activates M phase-specific transcription. Plant Cell. 1998;10(3):331. 
55. Gea G, Nathalie M, Anna G, Sundström JF, Lars R, Ines E. The RY/Sph element mediates transcriptional repression of maturation genes from late maturation to early seedling growth. New Phytol. 2010;184(3):552-65.

56. Hudson K. The circadian clock-controlled Transcriptome of developing soybean seeds. Plant Genom. 2010;3(1):3-13.

57. Kazumi N, Masanori O, Tomokazu K, Yuji K, Eiji N. Genome-wide profiling of stored mRNA in Arabidopsis thaliana seed germination: epigenetic and genetic regulation of transcription in seed. Plant J. 2010;41(5):697-709.

58. Kim E, Hwang S, Lee I. SoyNet: a database of co-functional networks for soybean Glycine max. Nucleic Acids Res. 2016;45(D1):D1082-9.

59. Visscher PM, Wray NR, Zhang Q, Sklar P, McCarthy Ml, Brown MA, Yang J. 10 years of GWAS discovery: biology, function, and translation. Am J Hum Genet. 2017;101(1):5-22.

60. Varshney RK, Sinha P, Singh VK, Kumar A, Zhang Q, Bennetzen JL. 5Gs for crop genetic improvement. Curr Opin Plant Biol. 2020;19:30119-0.

61. Xu Y, Yang T, Zhou Y, Yin S, Li P, Liu J, Xu S, Yang Z, Xu C. Genome-wide association mapping of starch pasting properties in maize using singlelocus and multi-locus models. Front Plant Sci. 2018;9:1311.

62. He L, Xiao J, Rashid KY, Yao Z, Li P, Jia G, Wang X, Cloutier S, You FM. Genome-wide association studies for pasmo resistance in flax (Linum usitatissimum L.). Front Plant Sci. 1982;2019:9.

63. Jeong N, Suh SJ, Kim M-H, Lee S, Moon J-K, Kim HS, Jeong S-C. Ln is a key regulator of leaflet shape and number of seeds per pod in soybean. Plant Cell. 2012;24(12):4807-18.

64. Lu X, Xiong Q, Cheng T, Li QT, Liu XL, Bi YD, Li W, Zhang WK, Ma B, Lai YC, Du WG. A PP2C-1 allele underlying a quantitative trait locus enhances soybean 100-seed weight. Mole Plant. 2017;10(5):670-68.

65. Zhao B, Dai A, Wei H, Yang S, Wang B, Jiang N, Feng X. Arabidopsis KLU homologue GmCYP78A72 regulates seed size in soybean. Plant Mole Biol. 2015:90(1-2):33-47.

66. Li N, Li Y. Ubiquitin-mediated control of seed size in plants. Front Plant Sci. 2014;5:332.

67. Li N, Li Y. Signaling pathways of seed size control in plants. Curr Opin Plant Biol. 2016:33:23-32.

68. Zhu X, Liang W, Cui X, Chen M, Yin C, Luo Z, Zhu J, Lucas WJ, Wang Z, Zhang D. Brassinosteroids promote development of rice pollen grains and seeds by triggering expression of carbon starved anther, a MYB domain protein. Plant J. 2015;82(4):570-81

69. Ren D, Wang X, Yang M, Yang L, He G, Deng XW. A new regulator of seed size control in Arabidopsis identified by a genome-wide association study. New Phytol. 2019;222(2):895-906.

70. Miao C, Wang D, He R, Liu S, Zhu JK. Mutations in MIR 396e and MIR $396 f$ increase grain size and modulate shoot architecture in rice. Plant Biotechnol J. 2020;18(2):491-501.

71. Liu E, Zeng S, Zhu S, Liu Y, Wu G, Zhao K, Liu X, Liu Q, Dong Z, Dang X, Xie $H$. Favorable alleles of GRAIN-FILLING RATE1 increase the grain-filling RATE and yield of Rice. Plant Physiol. 2019;181:1207-22.

72. Lafon-Placette C, Kōhler C. Embryo and endosperm, partners in seed development. Curr Opin Plant Biol. 2014;17:64-9.

73. D'Erfurth I, Signor CL, Aubert G, Sanchez M, Gallardo K. A role for an endosperm-localized subtilase in the control of seed size in legumes. New Phytol. 2012;196(3):738-51.

74. Zhu B, Hui L, Jiangqi W, S. MK, Xianbing W, Yanxi P, Lifang N, Hao L. Functional specialization of duplicated AGAMOUS homologs in regulating floral organ development of Medicago truncatula. Front Plant Sci. 2018;9: 854.

75. Yua F, Li J, Huang Y, Liu L, Li D, Chen L, Luan S. FERONIA receptor kinase controls seed size in Arabidopsis thaliana. Mole Plant. 2014;5(7):920-2.

76. Hills MJ. Control of storage-product synthesis in seeds. Curr Opin Plant Biol. 2004;7(3):302-8

77. Wang S, Yokosho K, Guo R, Whelan J, Ruan Y-L, Ma JF, Shou H. The soybean sugar transporter GmSWEET15 mediates sucrose export from endosperm to early embryo. Plant Physiol. 2019;180(4):2133-41.

78. Yang J, Luo D, Yang B, Frommer WB, Eom JS. SWEET 11 and 15 as key players in seed filling in rice. New Phytol. 2018;218(2):604-15.

79. Chen LQ, Lin IW, Qu X-Q, Sosso D, McFarlane HE, Londoño A, Samuels AL, Frommer WB. A Cascade of sequentially expressed sucrose transporters in the seed coat and endosperm provides nutrition for the Arabidopsis embryo. Plant Cell. 2015;27(3):607-19.

80. Tian S, Wu J, Liu Y, Huang X, Li F, Wang Z, Sun M-X. Ribosomal protein NtRPL17 interacts with kinesin-12 family protein NtKRP and functions in the regulation of embryo/seed size and radicle growth. J Exper Bot. 2017;68(20): 5553-64.

81. Kitagawa K, Kurinami S, Oki K, Abe Y, Ando T, Kono I, Yano M, Kitano H, Iwasaki Y. A novel Kinesin 13 protein regulating Rice seed length. Plant Cell Physiol. 2010;51(8):1315-29.

82. Wu T, Shen $Y$, Zheng $M$, Yang $C$, Chen $Y$, Feng $Z$, Liu $X$, Liu S, Chen Z, Lei $C$. Gene $S G L$, encoding a kinesin-like protein with transactivation activity, is involved in grain length and plant height in rice. Plant Cell Rep. 2014;33(2): 235-44.

83. Dewitte W, Scofield S, Alcasabas AA, Maughan SC, Menges M, Braun N, Collins C, Nieuwland J, Prinsen E, Sundaresan V, Murray JA. Arabidopsis CYCD3 D-type cyclins link cell proliferation and endocycles and are ratelimiting for cytokinin responses. Proc Natl Acad Sci U S A. 2007:104(36): 14537-42.

84. Schilling S, Pan S, Kennedy A, Melzer R. MADS-box genes and crop domestication: the jack of all traits. J Exper Bot. 2018;69(7):1447-69.

85. Pandey S, Reddy CS, Yaqoob U, Negi YK, Arora S, Kau T. In-silico analysis of Cis-acting regulatory elements (CAREs) in upstream regions of Ascorbate glutathione pathway genes from Oryza sativa. Bioch Physiol. 2015:4:159.

86. Fauteux F, Strömvik MV. Seed storage protein gene promoters contain conserved DNA motifs in Brassicaceae, Fabaceae and Poaceae. BMC Plant Biol. 2009;9(1):126-0.

87. Hintze J, Nelson R. Violin plots: a box plot-density trace synergism. Am Stat. 1998;52(2):181-4.

88. Nyquist WE, Baker RJ. Estimation of heritability and prediction of selection response in plant populations. Critical Rev Plant Sci. 1991;10(3):235-322.

89. Murray MG, Thompson WF. Rapid isolation of high molecular weight plant DNA. Nucleic Acids Res. 1980:8(19):4321-5.

90. Li L, Guo N, Niu J, Wang Z, Cui X, Sun J, Zhao T, Xing H. Loci and candidate gene identification for resistance to Phytophthora sojae via association analysis in soybean [Glycine max (L.) Merr.]. Mol Gen Genomics. 2016;291(3): 1095-103.

91. Schmutz J, Cannon SB, Schlueter J, Ma J, Mitros T, Nelson W, Hyten DL, Song Q, Thelen JJ, Cheng J. Genome sequence of the palaeopolyploid soybean. Nature. 2010;463(7278):178.

92. Li R, Yu C, Li Y, Lam TW, Yiu SM, Kristiansen K, Wang J. SOAP2: an improved ultrafast tool for short read alignment. Bioinformatics. 2009;25(15):1966-7.

93. Yi X, Liang $Y$, Huerta-Sanchez $E$, Jin X, Cuo ZXP, Pool JE, Xu X, Jiang H, Vinckenbosch N, Korneliussen TS, et al. Sequencing of 50 human Exomes reveals adaptation to high altitude. Science. 2010;329(5987):75-8.

94. Scheet P, Stephens M. A fast and flexible statistical model for large-scale population genotype data: applications to inferring missing genotypes and haplotypic phase. Am J Hum Genet. 2006;78(4):629-44.

95. LiLin Y. CMplot: Circle Manhattan Plot. 2019. Available at: https://CRAN.Rproject.org/package=CMplot. Accessed on 23 Nov 2019.

96. Alexander $\mathrm{DH}$, Novembre J, Lange K. Fast model-based estimation of ancestry in unrelated individuals. Genome Res. 2009:19(9):1655-64.

97. Evanno G, Regnaut S, Goudet J. Detecting the number of clusters of individuals using the software STRUCTURE: a simulation study. Mole Ecol. 2010;14(8):2611-20.

98. Bradbury P, Zhang Z, Kroon D, Casstevens T, Buckler EY. TASSEL: software for association mapping of complex traits in diverse samples. Bioinfor. 2007; 23(19):2633-5.

99. Lipka AE, Feng T, Qishan W, Jason P, Meng L, Bradbury PJ, Gore MA, Buckler ES, Zhiwu Z. GAPIT: genome association and prediction integrated tool. Bioinfor. 2012;28(18):2397-9.

100. Wang S-B, Feng J-Y, Ren W-L, Huang B, Zhou L, Wen Y-J, Zhang J, Dunwell JM, Xu S, Zhang Y-M. Improving power and accuracy of genome-wide association studies via a multi-locus mixed linear model methodology. Sci Rep. 2016;6:19444

101. Tamba CL, Zhang Y-M. A fast mrMLM algorithm for multi-locus genomewide association studies. BioRxiv. 2018:341784.

102. Zhang J, Feng J, Ni Y, Wen Y, Niu Y, Tamba C, Yue C, Song Q, Zhang Y. pLARmEB: integration of least angle regression with empirical Bayes for multilocus genome-wide association studies. Heredity. 2017;118(6):517.

103. Ren WL, Wen YJ, Dunwell JM, Zhang YM. pKWmEB: integration of KruskalWallis test with empirical Bayes under polygenic background control for multi-locus genome-wide association study. Heredity. 2018;120(3):208-18.

104. Tamba CL, Ni YL, Zhang YM. Iterative sure independence screening EMBayesian LASSO algorithm for multi-locus genome-wide association studies. PLoS Comput Biol. 2017;13(1):e1005357. 
105. McCouch SR, Chen X, Panaud O, Temnykh S, Xu Y, Yong GC, Huang N, Ishii T, Blair M. Microsatellite marker development, mapping and applications in rice genetics and breeding. Plant Mole Biol. 1997;35(1-2):89-99.

106. Barrett JC, Fry B, Maller J, Daly MJ. Haploview: analysis and visualization of LD and haplotype maps. Bioinfor. 2005;21(2):263-5.

107. Ning W, Akey JM, Kun Z, Ranajit C, Li J. Distribution of recombination crossovers and the origin of haplotype blocks: the interplay of population history, recombination, and mutation. Am J Hum Genet. 2002;71(5):1227-34

108. Grant D, Nelson RT, Cannon SB, Shoemaker RC. SoyBase, the USDA-ARS soybean genetics and genomics database. Nucleic Acids Res. 2010; 38(Database issue):D843.

109. Li J, Dai X, Liu T, Zhao PX. LegumelP: an integrative database for comparative genomics and transcriptomics of model legumes. Nucleic Acids Res. 2011:40(D1):D1221-9.

110. Gonzales MD, Eric A, Andrew F, Kamal G, David G, Randy S, Beavis WD, Waugh ME. The legume information system (LIS): an integrated information resource for comparative legume biology. Nucleic Acids Res. 2005; 33(Database issue):D660-5.

111. Lescot M, Patrice D, Gert T, Kathleen M, Yves M, Yves VDP, Pierre R, Stephane R. PlantCARE, a database of plant cis-acting regulatory elements and a portal to tools for in silico analysis of promoter sequences. Nucleic Acids Res. 2002;30(1):325-7.

112. Lopes CT, Max F, Farzana K, Donaldson SL, Quaid M, Bader GD. Cytoscape web: an interactive web-based network browser. Bioinfor. 2010;26(18):2347-8.

113. Zhou G, Soufan O, Ewald J, Hancock RE, Basu N, Xia J. NetworkAnalyst 3.0: a visual analytics platform for comprehensive gene expression profiling and meta-analysis. Nucleic Acids Res. 2019;47(W1):W234-41.

114. Fehr W, Caviness C, Burmood D, Pennington J. Stage of development descriptions for soybeans, Glycine max (L.) Merrill. Crop Sci. 1971;11(6):929-31.

\section{Publisher's Note}

Springer Nature remains neutral with regard to jurisdictional claims in published maps and institutional affiliations.

Ready to submit your research? Choose BMC and benefit from:

- fast, convenient online submission

- thorough peer review by experienced researchers in your field

- rapid publication on acceptance

- support for research data, including large and complex data types

- gold Open Access which fosters wider collaboration and increased citations

- maximum visibility for your research: over $100 \mathrm{M}$ website views per year

At $\mathrm{BMC}$, research is always in progress.

Learn more biomedcentral.com/submissions 